\title{
On the asymptotic expansion of certain plane singular integral operators
}

Vladimir Vasilyev ${ }^{*}$

\section{"Correspondence:}

vladimir.b.vasilyev@gmail.com

Chair of Differential Equations, Belgorod National Research

University, Studencheskaya 14/1, Belgorod, 308007, Russia

\section{Springer}

\begin{abstract}
We discuss the problem of the asymptotic expansion for some operators in a general theory of pseudo-differential equations on manifolds with borders. Using the distribution theory one obtains certain explicit representations for these operators. These limit distributions are constructed with the help of the Fourier transform, the Dirac mass-function and its derivatives, and the well-known distribution related to the Cauchy type integral.
\end{abstract}

Keywords: pseudo-differential operator; distribution; singularity; asymptotic expansion

\section{Introduction}

In the theory of pseudo-differential equations the main difficulty is studying model operators in canonical domains according to a local principle. It shows that for a Fredholm property of a general pseudo-differential operator on a compact manifold one needs the invertibility of its local representatives in each point of a manifold $[1,2]$. The author wrote many times on the nature of these local representatives, these are distinct in dependence on a point of a manifold. Each 'singularity' of a compact manifold (a half-space is a model situation for the smooth part of a boundary, cone for the conical point, wedge, etc.) corresponds to a certain distribution, and a convolution operator with this distribution describes a local representative of an initial pseudo-differential operator in a corresponding point of the manifold. All details can be found in [3-5]. But singularities can be of distinct dimensions and it is possible that such singularities of a low dimension can be obtained from analogous singularities of full dimension. This means we need to find distributions for limit cases when some of the parameters of the singularities tend to zero. This approach was partially realized in [6,7], and [5] is devoted to multi-dimensional constructions. Our idea is the following. Limiting operators for thin singularities obtained in [6] may be a zero approximation for such thin singularities. It is desirable to obtain an asymptotic expansion with a small parameter for the distribution corresponding to such a singularity. We will consider here a two-dimensional case and hope these studies will help us to transfer such constructions to multi-dimensional situations [5].

The theory of differential or more generally pseudo-differential equations and boundary value problems for manifolds with non-smooth boundaries includes now a lot of interesting approaches and results.

(c) The Author(s) 2017. This article is distributed under the terms of the Creative Commons Attribution 4.0 International License (http://creativecommons.org/licenses/by/4.0/), which permits unrestricted use, distribution, and reproduction in any medium, provided you give appropriate credit to the original author(s) and the source, provide a link to the Creative Commons license, and indicate if changes were made. 
Other approaches, technical tools and results in the theory of boundary value problems one can find in work of Mazya [8, 9], Plamenevskii [10], Schulze [11], Melrose [12-14], Taylor [15], Nistor [16], Dauge [17], Costabel [18], Mazzeo [19] and many others. We cannot enumerate all authors but in [3] a very large survey of these approaches is given.

The author wrote many times on another approach to studying solvability for pseudodifferential equations in domains with conical points and wedges, but now we would like to speak of the principal difference of our papers from other authors (Maz'ya, Plamenevski, Schulze and many others).

In all papers the conical domain is treated as a direct product of a circle and a half-axis (but in my point of view, it is a cylinder), then they apply the Mellin transform on the halfaxis, and the initial problem is reduced to a problem in a domain with a smooth boundary with operator-valued symbol. It follows further it is like the generalization of well-known results on the case of an operator symbol. Of course, our approach is a generalization also, but it is a generalization on dimension space, and the principal difference is that we do not divide the cone, and it is treated as an emergent thing.

For convenience of the reader the theory of the solvability for considered pseudodifferential equations, already known in principle [3, 4], is given in the next section of this paper.

\section{Solving pseudo-differential equations}

\subsection{Model operators and Sobolev-Slobodetskii spaces in a cone}

Pseudo-differential operators are locally defined by the well-known formula

$$
u(x) \longmapsto \int_{\mathbf{R}^{m}} \int_{\mathbf{R}^{m}} A(x, \xi) u(y) e^{i(x-y) \cdot \xi} d \xi d y, \quad x \in \mathbf{R}^{m},
$$

if $M$ is a compact smooth manifold because one can use the 'freezing coefficients principle' or, in other words, a 'local principle.' For a manifold with a smooth boundary we need a new local formula for defining the operator $A$ : more precisely near inner points of $M$ we use the usual formula, but near the boundary points we need another formula:

$$
u(x) \longmapsto \int_{\mathbf{R}_{+}^{m}} \int_{\mathbf{R}^{m}} A(x, \xi) u(y) e^{i(x-y) \cdot \xi} d \xi d y, \quad x \in \mathbf{R}_{+}^{m},
$$

where $\mathbf{R}_{+}^{m}=\left\{x \in \mathbf{R}^{m}: x=\left(x^{\prime}, x_{m}\right), x_{m}>0\right\}$.

For invertibility of such an operator with symbol $A(\cdot, \xi)$ not depending on a spatial variable $x$ one can apply the theory of the classical Riemann boundary value problems for upper and lower complex half-planes with a parameter $\xi^{\prime}=\left(\xi_{1}, \ldots, \xi_{m-1}\right)$. This step was systematically studied in [2]. But if the boundary $\partial M$ has at least one conical point, this approach is not effective.

A conical point at the boundary is such a point for which its neighborhood is diffeomorphic to the cone $C_{+}^{a}=\left\{x \in \mathbf{R}^{m}: x_{m}>a\left|x^{\prime}\right|, x^{\prime}=\left(x_{1}, \ldots, x_{m-1}\right), a>0\right\}$, hence a local definition for pseudo-differential operator near the conical point is the following:

$$
u(x) \longmapsto \int_{C_{+}^{a}} \int_{\mathbf{R}^{m}} A(x, \xi) u(y) e^{i(x-y) \cdot \xi} d \xi d y, \quad x \in C_{+}^{a} .
$$


To describe a solvability picture for the model elliptic pseudo-differential equation with the operator (1)

$$
(A u)(x)=v(x), \quad x \in C_{+}^{a},
$$

with symbol $A(\cdot, \xi)$ non-depending on a spatial variable $x$ in multi-dimensional cone $C_{+}^{a}=$ $\left\{x \in \mathbf{R}^{2}: x_{2}>a\left|x_{1}\right|, a>0\right\}$ earlier we considered the special singular integral operator [4]

$$
\left(K_{a} u\right)(x)=\frac{a}{2 \pi^{2}} \lim _{\tau \rightarrow 0+} \int_{\mathbf{R}^{2}} \frac{u(y) d y}{\left(x_{1}-y_{1}\right)^{2}-a^{2}\left(x_{2}-y_{2}+i \tau\right)^{2}} .
$$

This operator served a conical singularity in the general theory of boundary value problems for elliptic pseudo-differential equations on manifolds with a non-smooth boundary. The operator $K_{a}$ is a convolution operator, and the parameter $a$ is the size of an angle, $x_{2}>a\left|x_{1}\right|, a=\cot \alpha$.

To study the invertibility property for the operator (1) we have introduced the concept of the wave factorization for an elliptic symbol near a singular boundary point $[3,4]$ and using this property we have described Fredholm properties for equation (2). We use SobolevSlobodetskii spaces for studying these properties.

Definition 1 By definition the Sobolev-Slobodetskii space $H_{s}\left(\mathbf{R}^{m}\right)$ consists of distributions $u$ for which their Fourier transforms are locally integrable functions $\tilde{u}(\xi)$ such that

$$
\|u\|_{s}^{2}=\int_{\mathbf{R}^{m}}|\tilde{u}(\xi)|^{2}(1+|\xi|)^{2 s} d \xi<+\infty, \quad \tilde{u}(\xi)=\int_{\mathbf{R}^{m}} u(x) e^{-i x \cdot \xi} d x
$$

We will denote the Fourier image of the space $H_{s}\left(\mathbf{R}^{m}\right)$ by $\tilde{H}_{s}\left(\mathbf{R}^{m}\right) . \tilde{H}_{s}\left(\mathbf{R}^{m}\right)$ and, consequently, $H_{s}\left(\mathbf{R}^{m}\right)$ are Hilbert spaces with respect to the inner product

$$
\langle u, v\rangle_{s}=\int_{\mathbf{R}^{m}} \tilde{u}(\xi) \overline{\tilde{v}(\xi)}(1+|\xi|)^{2 s} d \xi
$$

and formula (3) defines the norm in the spaces $H_{s}\left(\mathbf{R}^{m}\right)$ and $\tilde{H}_{s}\left(\mathbf{R}^{m}\right)$.

If $s=0$ then $\tilde{H}_{0}\left(\mathbf{R}^{m}\right)=L_{2}\left(\mathbf{R}^{m}\right)$, and by virtue of Plancherel's theorem $H_{0}\left(\mathbf{R}^{m}\right)=$ $F^{-1} \tilde{H}_{0}\left(\mathbf{R}^{m}\right)=L_{2}\left(\mathbf{R}^{m}\right)$.

In the case $s=n$ ( $n>0, n$ integer) $H_{n}\left(\mathbf{R}^{m}\right)$ consists of functions $u(x)$ that are integrable with their square functions, for which their generalized derivatives $\partial D^{k} u(x)$ under $1 \leq|k| \leq n$ are integrable with their square functions also. The norm (3) in this case is equivalent to the following norm:

$$
\|u\|_{n}^{2}=\sum_{|k| \leq n} \int_{\mathbf{R}^{m}}\left|\partial D^{k} u(x)\right|^{2} d x=\sum_{|k| \leq n} \frac{1}{(2 \pi)^{m}} \int_{\mathbf{R}^{m}}\left|\xi^{k} \tilde{u}(\xi)\right|^{2} d \xi
$$

In the case $s=-n, n>0, n$ integer, the distributions from $H_{-n}\left(\mathbf{R}^{m}\right)$ are derivatives of functions from $L_{2}\left(\mathbf{R}^{m}\right)$ whose order is not higher than $n$.

By definition, the space $H_{s}\left(C_{+}^{a}\right)$ consists of distributions from $H_{s}\left(\mathbf{R}^{m}\right)$, which support belongs to $\overline{C_{+}^{a}}$. The norm in the space $H_{s}\left(C_{+}^{a}\right)$ is induced by the norm from $H_{s}\left(\mathbf{R}^{m}\right)$. The 
right-hand side $v$ is chosen from the space $H_{s-\alpha}^{0}\left(C_{+}^{a}\right)$, which is the space of distributions $S^{\prime}\left(C_{+}^{a}\right)$, admitting the continuation on $H_{s-\alpha}\left(\mathbf{R}^{m}\right)$. The norm in the space $H_{s-\alpha}^{0}\left(C_{+}^{a}\right)$ is defined

$$
\|v\|_{s-\alpha}^{+}=\inf \|l v\|_{s-\alpha},
$$

where the infimum is chosen from all continuations $l$.

\subsection{Wave factorization and solvability}

Let us return to equation (2). We will recall some of our preliminary results [3, 4]. The symbol $\stackrel{*}{C_{+}^{a}}$ denotes a conjugate cone for $C_{+}^{a}$ :

$$
\stackrel{*}{C_{+}^{a}}=\left\{x \in \mathbf{R}^{2}: x=\left(x_{1}, x_{2}\right), a x_{2}>\left|x_{1}\right|\right\}
$$

$C_{-}^{a} \equiv-C_{+}^{a}, T\left(C_{+}^{a}\right)$ denotes a radial tube domain over the cone $C_{+}^{a}[20]$, i.e. the domain in a complex space $\mathbf{C}^{2}$ of type $\mathbf{R}^{2}+i C_{+}^{a}$.

We consider symbols $A(\xi)$ satisfying the condition

$$
c_{1} \leq\left|A(\xi)(1+|\xi|)^{-\alpha}\right| \leq c_{2},
$$

which are elliptic, and the number $\alpha \in \mathbf{R}$ is called an order of the operator $A$.

To describe the solvability picture for equation (2) we use the following.

Definition 2 Wave factorization with respect to the cone $C_{+}^{a}$ for the symbol $A(\xi)$ is called a representation in the form

$$
A(\xi)=A_{\neq}(\xi) A_{=}(\xi)
$$

where the factors $A_{\neq}(\xi), A_{=}(\xi)$ must satisfy the following conditions:

(1) $A_{\neq}(\xi), A_{=}(\xi)$ are defined for all admissible values $\xi \in \mathbf{R}^{2}$, without maybe the points $\left\{\xi \in \mathbf{R}^{2}:\left|\xi_{1}\right|^{2}=a^{2} \xi_{2}^{2}\right\}$

(2) $A_{\neq}(\xi), A_{=}(\xi)$ admit an analytical continuation into radial tube domains $T\left(\stackrel{*}{C_{+}^{a}}\right)$, $T\left(\stackrel{*}{C_{-}^{a}}\right)$, respectively, with estimates

$$
\begin{aligned}
& \left|A_{\neq}^{ \pm 1}(\xi+i \tau)\right| \leq c_{1}(1+|\xi|+|\tau|)^{ \pm \kappa}, \\
& \left|A_{=}^{ \pm 1}(\xi-i \tau)\right| \leq c_{2}(1+|\xi|+|\tau|)^{ \pm(\alpha-\kappa)}, \forall \tau \in C_{+}^{*} .
\end{aligned}
$$

The number $\kappa \in \mathbf{R}$ is called the index of the wave factorization.

For $|\kappa-s|<1 / 2$ one has the existence and uniqueness theorem [3]. For this purpose we need a certain lemma.

Lemma 1 Let functions $B_{\neq}(\xi+i \tau), B_{=}(\xi+i \tau)$ be analytical in $T\left(\stackrel{*}{C_{+}^{a}}\right)$ and $T\left(\stackrel{*}{C_{-}^{a}}\right)$ and satisfy the estimates

$$
\begin{array}{ll}
\left|B_{7}(\xi+i \tau)\right| \leq c_{1}(1+|\xi|+|\tau|)^{\alpha}, & \tau \in C_{+}^{*}, \\
\left|B_{=}(\xi+i \tau)\right| \leq c_{2}(1+|\xi|+|\tau|)^{\alpha}, & \tau \in C_{-}^{a}
\end{array}
$$


Then the multiplication operator by the function $B_{7}(\xi)$ boundedly acts from space $\tilde{H}_{s}\left(C_{+}^{a}\right)$ into $\tilde{H}_{s-\alpha}\left(C_{+}^{a}\right)$, and the multiplication operator by the function $B_{=}(\xi)$ from space $\tilde{H}_{s}\left(\mathbf{R}^{2} \backslash \overline{C_{+}^{a}}\right)$ into space $\tilde{H}_{s-\alpha}\left(\mathbf{R}^{2} \backslash \overline{C_{+}^{a}}\right)$.

Proof The fact that multiplication operators by functions $B_{\neq}(\xi), B_{=}(\xi)$ boundedly act from spaces $\tilde{H}_{s}\left(C_{+}^{a}\right), \tilde{H}_{s}\left(\mathbf{R}^{2} \backslash \overline{C_{+}^{a}}\right)$ into space $\tilde{H}_{s-\alpha}\left(\mathbf{R}^{2}\right)$ is well known [2]. For clarity we denote $u \in \tilde{H}_{s}\left(C_{+}^{a}\right)$ by $u_{+}$and $u \in \tilde{H}_{s}\left(\mathbf{R}^{2} \backslash \overline{C_{+}^{a}}\right)$ by $u_{-}$. Let us show that $B_{\neq}(\xi) \tilde{u}_{+}(\xi) \in \tilde{H}_{s-\alpha}\left(C_{+}^{a}\right)$ for any $\tilde{u}_{+} \in \tilde{H}_{s}\left(C_{+}^{a}\right)$.

The space $\tilde{H}_{s}\left(C_{+}^{a}\right)$ has an explicit description [3]: $\tilde{u}_{+} \in \tilde{H}_{s}\left(C_{+}^{a}\right)$ if and only if $\tilde{u}_{+}(\xi+i \tau)$ is analytical in $T\left(\stackrel{*}{C}_{+}^{a}\right)$ and the quantity

$$
\sup \int_{\mathbf{R}^{2}}\left|\tilde{u}_{+}(\xi+i \tau)\right|^{2}(1+|\xi|)^{2 s} d \xi, \quad \tau \in C_{+}^{*}
$$

is finite and coincides with

$$
\int_{\mathbf{R}^{2}}\left|\tilde{u}_{+}(\xi)\right|^{2}(1+|\xi|)^{2 s} d \xi
$$

Then evidently, $B_{\neq}(\xi+i \tau) \tilde{u}_{+}(\xi+i \tau)$ is analytical in $T\left(\stackrel{*}{C_{+}^{a}}\right)$ and

$$
\begin{aligned}
& \sup _{\substack{\xi \in C_{+}^{a} \\
\tau \mathbf{R}^{2}}} \int_{\neq}\left|B_{\neq}(\xi+i \tau) \tilde{u}_{+}(\xi+i \tau)\right|^{2}(1+|\xi|)^{2(s-\alpha)} d \xi \\
& \quad=\int_{\mathbf{R}^{2}}\left|B_{\neq}(\xi) \tilde{u}_{+}(\xi)\right|^{2}(1+|\xi|)^{2(s-\alpha)} d \xi \leq c \int_{\mathbf{R}^{2}}|\tilde{u}(\xi)|^{2}(1+|\xi|)^{2 s} d \xi
\end{aligned}
$$

i.e., $B_{7}(\xi) \tilde{u}_{+}(\xi) \in \tilde{H}_{s-\alpha}\left(C_{+}^{a}\right){ }^{\mathrm{a}}$

Now let us consider $B_{=}(\xi) \tilde{u}_{-}(\xi)$. Let at first $u_{-} \in C_{0}^{\infty}\left(\mathbf{R}^{2} \backslash \overline{C_{+}^{a}}\right)$. Of course $F^{-1} B_{=} \equiv b$ exists in the distribution sense and $\operatorname{supp} b \subset-\overline{C_{+}^{a}}$, as above. Then $F^{-1}\left(B_{-} \tilde{u}_{-}\right)=b * u_{-}$. By the definition of a convolution

$$
\left(b * u_{-}\right)(x)=\left(b(y), \overline{u_{-}(x-y)}\right),
$$

where $\overline{u_{-}(x-y)}$ is considered as a function on $y$ ( $x$ is fixed), and notation $b(y)$ means that functional $b$ acts on $y$, a variable. Let us show that $\left(b * u_{-}\right)(x)=0$ under $x \in C_{+}^{a}$. Consider two cases: $y \in-C_{+}^{a}$ and $y \notin-C_{+}^{a}$. In the first case $x-y \in C_{+}^{a}$ and, thus $u_{-}(x-y)=0$ because $\operatorname{supp} u_{-}(x-y) \subset \mathbf{R}^{2} \backslash C_{+}^{a}$. In the second case $\left(b * u_{-}\right)$vanishes because $y \notin \operatorname{supp} b$.

Transfer to the general case $u_{-} \in H_{s}\left(\mathbf{R}^{2} \backslash \overline{C_{+}^{a}}\right)$ is realized by virtue of the density of class $C_{0}^{\infty}\left(\mathbf{R}^{2} \backslash \overline{C_{+}^{a}}\right)$ in space $H_{s}\left(\mathbf{R}^{2} \backslash \overline{C_{+}^{a}}\right)$.

So, it was shown that $B_{=}(\xi) \tilde{u}_{-}(\xi) \in \tilde{H}_{s-\alpha}\left(\mathbf{R}^{2}\right)$ and $\operatorname{supp} F^{-1}\left(B_{=} \tilde{u}_{-}\right) \subset\left(\mathbf{R}^{2} \backslash C_{+}^{a}\right)$. Hence, $B_{=}(\xi) \tilde{u}_{-}(\xi) \in \tilde{H}_{s-\alpha}\left(\mathbf{R}^{2} \backslash \overline{C_{+}^{a}}\right)$.

As above we use the notation $u_{+}$for the function $u \in H_{s}\left(C_{+}^{a}\right)$.

Theorem 1 If the elliptic symbol $A(\xi)$ admits wave factorization with respect to the cone $C_{+}^{a}$ and $|\kappa-s|<1 / 2$, then equation (2) has a unique solution $u_{+} \in H_{s}\left(C_{+}^{a}\right)$ for an arbitrary 
right-hand side $v \in H_{s-\alpha}^{0}\left(C_{+}^{a}\right)$,

$$
\tilde{u}_{+}(\xi)=A_{\neq}^{-1}(\xi)\left(K_{a} \widetilde{l v}\right)(\xi)
$$

where lv is an arbitrary continuation of $v$ on the whole $H_{s-\alpha}\left(\mathbf{R}^{2}\right)$.

A priori estimate holds:

$$
\left\|u_{+}\right\|_{s} \leq c\|v\|_{s-\alpha}^{+} .
$$

Proof We give here this proof to explain the appearance of the operator $K_{a}$ because it plays a crucial role in our studies.

Let us denote

$$
u_{-}=l v-A u_{+} .
$$

Taking into account wave factorization after applying to (4) the Fourier transform we have

$$
A_{\neq}(\xi) \tilde{u}_{+}(\xi)+A_{=}^{-1}(\xi) \tilde{u}_{-}(\xi)=A_{=}^{-1}(\xi) \tilde{l} v(\xi) .
$$

According to the properties of wave factorization elements $A_{\neq}(\xi), A_{=}(\xi)$ we have $A_{\ddagger}(\xi) \tilde{u}_{+}(\xi) \in \tilde{H}_{s-\kappa}\left(C_{+}^{a}\right), A_{=}^{-1}(\xi) \tilde{u}_{-}(\xi) \in \tilde{H}_{s-\kappa}\left(\mathbf{R}^{2} \backslash \overline{C_{+}^{a}}\right)$ (because $\tilde{u}_{-} \in \tilde{H}_{s-\alpha}\left(\mathbf{R}^{2} \backslash \overline{C_{+}^{a}}\right)$ ), $A_{=}^{-1}(\xi) \tilde{l} v(\xi) \in \tilde{H}_{s-\kappa}\left(\mathbf{R}^{2}\right)$, where $\kappa$ is index of wave factorization. Since $|s-\kappa|<1 / 2, \tilde{H}_{s-\kappa}\left(\mathbf{R}^{2}\right)$ admits a unique representation as a sum of two orthogonal subspaces $\tilde{H}_{s-\kappa}\left(C_{+}^{a}\right)$ and $\tilde{H}_{s-\kappa}\left(\mathbf{R}^{2} \backslash \overline{C_{+}^{a}}\right)[3]$ so that

$$
A_{\neq} \tilde{u}_{+}=K_{a} A_{=}^{-1} \tilde{l}
$$

and it implies

$$
\tilde{u}_{+}=A_{\ddagger}^{-1} K_{a} A_{=}^{-1} \tilde{l} \text {. }
$$

A priori estimate is

$$
\begin{aligned}
\left\|u_{+}\right\|_{s} & =\left\|\tilde{u}_{+}\right\|_{s} \leq c\left\|K_{a} A_{=}^{-1} \tilde{l v}\right\|_{s-\kappa} \\
& \leq c\left\|A_{=}^{-1} \tilde{l v}\right\|_{s-\kappa} \leq c\|\tilde{l}\|_{s-\kappa} \\
& =c\|\tilde{l}\|_{s-\alpha} \leq c\|v\|_{s-\alpha}^{+},
\end{aligned}
$$

taking into account boundedness of operator $K_{a}$ in $\tilde{H}_{s}\left(\mathbf{R}^{2}\right)$ for $|s|<1 / 2$ and boundedness of continuation operator $l[3]$.

Remark 1 If $|\kappa-s|>1 / 2$ there are additional conditions or solvability conditions for the right-hand side to obtain a unique solvability for equation (2) in appropriate SobolevSlobodetskii spaces [3]. 


\section{An initial approximation}

The operator $K_{a}$ can be treated as a convolution operator with the following distribution:

$$
\frac{a}{2 \pi^{2}} \frac{1}{\xi_{1}^{2}-a^{2} \xi_{2}^{2}}
$$

and starting from this assertion we will work with this distribution taking into account its relationship with the operator $K_{a}$.

We will consider two spaces of basic functions for distributions. If $D\left(\mathbf{R}^{2}\right)$ denotes a space of infinitely differentiable functions with a compact support then $D^{\prime}\left(\mathbf{R}^{2}\right)$ is the corresponding space of distributions over the space $D\left(\mathbf{R}^{2}\right)$; analogously if $S\left(\mathbf{R}^{2}\right)$ is the Schwartz space of functions infinitely differentiable rapidly decreasing at infinity, then $S^{\prime}\left(\mathbf{R}^{2}\right)$ is a corresponding space of distributions over $S\left(\mathbf{R}^{2}\right)$.

When $a \rightarrow+\infty$ one obtains [6] the following limit distribution:

$$
\lim _{a \rightarrow \infty} \frac{a}{2 \pi^{2}} \frac{1}{\xi_{1}^{2}-a^{2} \xi_{2}^{2}}=\frac{i}{2 \pi} \mathcal{P} \frac{1}{\xi_{1}} \otimes \delta\left(\xi_{2}\right)
$$

where the notation for distribution $\mathcal{P}$ is taken from Vladimirov's work [20, 21], and $\otimes$ denotes the direct product of distributions. Here $\delta$ denotes one-dimensional Dirac massfunction, which acts on $\varphi \in D(\mathbf{R})$ in the following way:

$$
(\delta, \varphi)=\varphi(0),
$$

and the distribution $\mathcal{P} \frac{1}{x}$ is defined by the formula

$$
\left(\mathcal{P} \frac{1}{x}, \varphi\right)=\text { v.p. } \int_{-\infty}^{+\infty} \frac{\varphi(x) d x}{x} \equiv \lim _{\varepsilon \rightarrow 0+}\left(\int_{-\infty}^{-\varepsilon}+\int_{\varepsilon}^{+\infty}\right) \frac{\varphi(x) d x}{x} .
$$

Let us note that the distribution (5) corresponds to the operator (see, for example [2])

$$
u(\xi) \longmapsto \frac{i}{2 \pi} \mathrm{v} \cdot \mathrm{p} \cdot \int_{-\infty}^{+\infty} \frac{u\left(\eta_{1}, \xi_{2}\right) d \eta_{1}}{\xi_{1}-\eta_{1}} .
$$

Our main goal in this paper is obtaining an asymptotical expansion for the twodimensional distribution

$$
K_{a}\left(\xi_{1}, \xi_{2}\right) \equiv \frac{a}{2 \pi^{2}} \frac{1}{\xi_{1}^{2}-a^{2} \xi_{2}^{2}}
$$

with respect to small $a^{-1}$. It is defined by the corresponding formula $\forall \varphi \in D\left(\mathbf{R}^{2}\right)$

$$
\left(K_{a}, \varphi\right)=\frac{a}{2 \pi^{2}} \int_{\mathbf{R}^{2}} \frac{\varphi\left(\xi_{1}, \xi_{2}\right) d \xi}{\xi_{1}^{2}-a^{2} \xi_{2}^{2}} .
$$

\section{A decomposition formula for distributions}

We will use the standard Maclaurin formula

$$
\varphi\left(\xi_{1}, \xi_{2}\right)=\sum_{k=0}^{\infty} \frac{\varphi_{\xi_{2}}^{(k)}\left(\xi_{1}, 0\right)}{k !} \xi_{2}^{k}
$$


and make the change of a variable $a \xi_{2}=t, a^{-1}=b$, then formula (6) will become

$$
\left(K_{a}, \varphi\right)=\frac{1}{2 \pi^{2}} \int_{\mathbf{R}^{2}} \frac{\varphi\left(\xi_{1}, b t\right) d \xi_{1} d t}{\xi_{1}^{2}-t^{2}}
$$

Remark 2 In [6] the author has considered the two cases $a \rightarrow \infty$ and $a \rightarrow 0$; the first case corresponds to a zero angle but the second one corresponds to a half-space; the last was done for a comparison with [2]. Since the half-space case is studied in [2] in detail we do not stop in this here.

Then we represent $\mathbf{R}^{2}=M \cup\left(\mathbf{R}^{2} \backslash M\right)$ where $M$ is a square with a line size $N$, so we have

$$
\left(K_{a}, \varphi\right)=\frac{1}{2 \pi^{2}}\left(\int_{M}+\int_{\mathbf{R}^{2} \backslash M}\right) \frac{\varphi\left(\xi_{1}, b t\right) d \xi_{1} d t}{\xi_{1}^{2}-t^{2}} .
$$

\subsection{A rough decomposition}

Let us consider here $\varphi \in D\left(\mathbf{R}^{2}\right)$. Since the support of $\varphi$ is a compact set we have one summand in formula (7); therefore we might work with the formula

$$
\frac{1}{2 \pi^{2}} \int_{\mathbf{R}^{2}} \frac{\varphi\left(\xi_{1}, b t\right) d \xi_{1} d t}{\xi_{1}^{2}-t^{2}}
$$

immediately.

More naturally it will be to proceed in the following way using a Maclaurin series:

$$
\left(K_{a}, \varphi\right)=\frac{1}{2 \pi^{2}} \sum_{k=0}^{\infty} \frac{1}{k !} \int_{\mathbf{R}^{2}} \frac{\varphi_{\xi_{2}}^{(k)}\left(\xi_{1}, 0\right) b^{k} t^{k} d \xi_{1} d t}{\xi_{1}^{2}-t^{2}} .
$$

If $t$ varies in a line segment then $b t \sim b, b \rightarrow 0$, and we can use the following formal representations [7]:

$$
K_{a}\left(\xi_{1}, \xi_{2}\right)=\frac{i}{2 \pi} \sum_{n=0}^{+\infty} \frac{(-1)^{n}}{n ! a^{n}} \mathcal{P} \frac{1}{\xi_{1}} \otimes \delta^{(n)}\left(\xi_{2}\right)
$$

\subsection{A sharp decomposition}

Here we consider $\varphi \in S\left(\mathbf{R}^{2}\right)$.

A formal use of the Maclaurin formula for the first integral in (7) will lead to the following result:

$$
\left(K_{a}, \varphi\right)=\frac{1}{2 \pi^{2}} \sum_{k=0}^{\infty} \frac{b^{k}}{k !} \int_{-N}^{+N} \varphi_{\xi_{2}}^{(k)}\left(\xi_{1}, 0\right)\left(\int_{-N}^{+N} \frac{t^{k} d t}{\xi_{1}^{2}-t^{2}}\right) d \xi_{1},
$$

and we need to give a certain meaning to the expression in brackets.

Let us denote

$$
T_{k, N}\left(\xi_{1}\right) \equiv \int_{-N}^{+N} \frac{t^{k} d t}{\xi_{1}^{2}-t^{2}}
$$

and reproduce some calculations. 
First $T_{k, N}\left(\xi_{1}\right) \equiv 0, \forall k=2 n-1, n \in \mathbf{N}$. So the non-trivial case is $k=2 n, n \in \mathbf{N}$. Let us recall $T_{0, \infty}\left(\xi_{1}\right)=\pi i 2^{-1} \xi_{1}^{-1}[6,7]$. For other cases we can calculate this integral so we have the following:

$$
\begin{aligned}
k= & 2, \\
& T_{2, N}\left(\xi_{1}\right)=-2 N-2^{-1} \xi_{1}^{-1} \ln \frac{N-\xi_{1}}{N+\xi_{1}}+\pi i 2^{-1} \xi_{1}^{-1} \\
k= & 4 \\
& T_{4, N}\left(\xi_{1}\right)=-2 / 3 N^{3}-2 \xi_{1}^{2} N-2^{-1} \xi_{1}^{3} \ln \frac{N-\xi_{1}}{N+\xi_{1}}+\pi i 2^{-1} \xi_{1}^{3} ; \\
k= & 6, \\
& T_{6, N}\left(\xi_{1}\right)=-2 / 5 N^{5}-2 / 3 \xi_{1}^{2} N^{3}-2 \xi_{1}^{5} N-2^{-1} \xi_{1}^{5} \ln \frac{N-\xi_{1}}{N+\xi_{1}}+\pi i 2^{-1} \xi_{1}^{5},
\end{aligned}
$$

and so on. One can easily write all expressions for arbitrary $T_{2 n, N}\left(\xi_{1}\right)$.

In general one can write

$$
T_{2 n, N}\left(\xi_{1}\right)=P_{2 n-1}\left(N, \xi_{1}\right)-2^{-1} \xi_{1}^{2 n-1} \ln \frac{N-\xi_{1}}{N+\xi_{1}}+\pi i 2^{-1} \xi_{1}^{2 n-1}
$$

where $P_{2 n-1}\left(N, \xi_{1}\right)$ is a certain polynomial of order $2 n-1$ on variables $N, \xi_{1}$.

Therefore instead of formula (8) we can write

$$
\begin{aligned}
\left(K_{a}, \varphi\right)= & \frac{i}{2 \pi}\left(\mathcal{P} \frac{1}{\xi_{1}} \otimes \delta\left(\xi_{2}\right), \varphi\right) \\
& +\frac{1}{2 \pi^{2}} \sum_{n=1}^{\infty} \frac{b^{2 n}}{(2 n) !} \int_{-N}^{+N} \varphi_{\xi_{2}}^{(2 n)}\left(\xi_{1}, 0\right) \\
& \times\left(P_{2 n-1}\left(N, \xi_{1}\right)-2^{-1} \xi_{1}^{2 n-1} \ln \frac{N-\xi_{1}}{N+\xi_{1}}+\pi i 2^{-1} \xi_{1}^{2 n-1}\right) d \xi_{1}
\end{aligned}
$$

Let us describe the polynomial $P_{2 n-1}\left(N, \xi_{1}\right)$ more precisely. Obviously

$$
P_{2 n-1}\left(N, \xi_{1}\right)=c_{2 n-1} N^{2 n-1}+c_{2 n-3} N^{2 n-3} \xi_{1}^{2}+\cdots+c_{1} N \xi_{1}^{2 n-1}
$$

Further we rewrite the equality (9) in the following form:

$$
\begin{aligned}
\left(K_{a}, \varphi\right)= & \frac{i}{2 \pi}\left(\mathcal{P} \frac{1}{\xi_{1}} \otimes \delta\left(\xi_{2}\right), \varphi\right) \\
& +\frac{1}{2 \pi^{2}} \sum_{n=1}^{\infty} \frac{b^{2 n}}{(2 n) !} \sum_{k=1}^{n} c_{2 k-1} N^{2 k-1} \int_{-N}^{+N} \varphi_{\xi_{2}}^{(2 n)}\left(\xi_{1}, 0\right) \xi_{1}^{2 k-1} d \xi_{1} \\
& -\frac{1}{4 \pi^{2}} \sum_{n=1}^{\infty} \frac{b^{2 n}}{(2 n) !} \int_{-N}^{+N} \varphi_{\xi_{2}}^{(2 n)}\left(\xi_{1}, 0\right) \xi_{1}^{2 n-1} \ln \frac{N-\xi_{1}}{N+\xi_{1}} d \xi_{1} \\
& +\frac{i}{4 \pi} \sum_{n=1}^{\infty} \frac{b^{2 n}}{(2 n) !} \int_{-N}^{+N} \varphi_{\xi_{2}}^{(2 n)}\left(\xi_{1}, 0\right) \xi_{1}^{2 n-1} d \xi_{1} .
\end{aligned}
$$


We will start from two last summands. The second summand does not play any role because

$$
\lim _{N \rightarrow+\infty} \ln \frac{N-\xi_{1}}{N+\xi_{1}}=0 .
$$

The third summand we will represent according to Lemma 2 (see below) taking into account that we can pass to the limit under $N \rightarrow+\infty$,

$$
\frac{i}{4 \pi} \sum_{n=1}^{\infty} \frac{b^{2 n}}{(2 n) !}\left(\widetilde{\delta^{(2 n-1)}}\left(\xi_{1}\right) \otimes \delta^{(2 n)}\left(\xi_{2}\right), \varphi\right) .
$$

For the first summand we consider separately the case $N b \sim 1(N \rightarrow \infty, b \rightarrow 0)$. In other words we consider a special limit to justify the decomposition. Then

$$
\begin{aligned}
& \frac{1}{2 \pi^{2}} \sum_{n=1}^{\infty} \frac{b^{2 n}}{(2 n) !} \sum_{k=1}^{n} c_{2 k-1} N^{2 k-1} \int_{-N}^{+N} \varphi_{\xi_{2}}^{(2 n)}\left(\xi_{1}, 0\right) \xi_{1}^{2 k-1} d \xi_{1} \\
& \quad \sim \frac{1}{2 \pi^{2}} \sum_{n=1}^{\infty} \frac{1}{(2 n) !} \sum_{k=1}^{n} c_{2 k-1} b^{2 n-2 k+1} \int_{-\infty}^{+\infty} \varphi_{\xi_{2}}^{(2 n)}\left(\xi_{1}, 0\right) \xi_{1}^{2 k-1} d \xi_{1} .
\end{aligned}
$$

Therefore

$$
\begin{aligned}
& \frac{1}{2 \pi^{2}} \sum_{n=1}^{\infty} \frac{1}{(2 n) !} \sum_{k=1}^{n} c_{2 k-1} b^{2 n-2 k+1} \int_{-\infty}^{+\infty} \varphi_{\xi_{2}}^{(2 n)}\left(\xi_{1}, 0\right) \xi_{1}^{2 k-1} d \xi_{1} \\
& \left.=\frac{1}{2 \pi^{2}} \sum_{n=1}^{\infty} \frac{1}{(2 n) !} \sum_{k=1}^{n} c_{2 k-1} b^{2 n-2 k+1} \widetilde{\left(\delta^{(2 k-1)}\right.}\left(\xi_{1}\right) \otimes \delta^{(2 n)}\left(\xi_{2}\right), \varphi\right) .
\end{aligned}
$$

One can note if desirable

$$
c_{2 k-1}=-2\left(1+\frac{1}{3}+\cdots+\frac{1}{2 k-1}\right) .
$$

\section{A local behavior of a boundary operator}

Lemma 2 If a distribution a acts on the function $\varphi \in S(\mathbf{R})$ in the following way:

$$
(a, \varphi)=\int_{-\infty}^{+\infty} \xi^{k} \varphi(\xi) d \xi
$$

then this distribution a is the following:

$$
a(\xi)=\widetilde{\delta^{(k)}}(\xi)
$$

where the sign $\sim$ means here the inverse Fourier transform $F^{-1}$.

Proof Indeed, we have $F \delta=\mathbf{1}$, where $\mathbf{1}$ is an identity in a distribution sense so that $F^{-1} \mathbf{1}=\delta$. Since

$$
\left(F\left(\varphi^{(k)}\right)\right)(\xi)=(-1)^{k} \xi^{k} \tilde{\varphi}(\xi)
$$


denoting $\psi=F^{-1} \varphi$ we write

$$
\begin{aligned}
(a, \varphi) & =(a, F \psi)=\int_{-\infty}^{+\infty} \xi^{k} \widetilde{\psi}(\xi) d \xi=\left(\mathbf{1}, \xi^{k} \widetilde{\psi}(\xi)\right)=\left(\mathbf{1}, F F^{-1}(\xi k \widetilde{\psi}(\xi))\right) \\
& =\left(F \mathbf{1}, F^{-1}\left(\xi_{k} \widetilde{\psi}(\xi)\right)\right)=\left(F \mathbf{1},(-1)^{k} \psi^{(k)}(x)\right)=\left(\delta,(-1)^{k} \psi^{(k)}(x)\right) \\
& =\left(\delta^{(k)}, \psi\right)=\left(\delta^{(k)}, F^{-1} \varphi\right)=\left(F^{-1} \delta^{(k)}, \varphi\right),
\end{aligned}
$$

so we have the required identity.

Theorem 2 The following formula:

$$
K_{a}\left(\xi_{1}, \xi_{2}\right)=\frac{i}{2 \pi} \mathcal{P} \frac{1}{\xi_{1}} \otimes \delta\left(\xi_{2}\right)+\sum_{m, n} c_{m, n}(a) \widetilde{\delta^{(m)}}\left(\xi_{1}\right) \otimes \delta^{(n)}\left(\xi_{2}\right)
$$

where $c_{m, n}(a) \rightarrow 0, a \rightarrow+\infty$, holds in a distribution sense.

Proof Returning to formula (8) and using calculations $T_{k, N}\left(\xi_{1}\right)$ and Lemma 2 we obtain the required assertion.

Remark 3 One can easily reconstruct the coefficients $c_{m, n}(a)$ starting from the above calculations.

\section{Towards a pseudo-differential equation}

Let us return to equation (2). For $|\kappa-s|<1 / 2$ one has the existence and uniqueness theorem [3]

$$
\widetilde{u}(\xi)=A_{\neq}^{-1}(\xi)\left(K_{a} \tilde{l}\right)(\xi)
$$

where $l v$ is an arbitrary continuation of $v$ on the whole $H_{s}\left(\mathbf{R}^{2}\right)$.

Below we denote $l v \equiv V$.

Theorem 3 If the symbol $A(\xi)$ admits the wave factorization with respect to the cone $C_{+}^{a}$ and $|\kappa-s|<1 / 2$ then equation (2) has a unique solution in the space $H^{s}\left(C_{+}^{a}\right)$, and for the large ' $a$ ' it can be represented in the form

$$
\begin{aligned}
\widetilde{u}(\xi)= & \frac{i}{2 \pi} A_{\ddagger}^{-1}(\xi) \mathrm{v} \cdot \mathrm{p} \cdot \int_{-\infty}^{+\infty} \frac{\left(A_{=}^{-1} \widetilde{V}\right)\left(\eta_{1}, \xi_{2}\right) d \eta_{1}}{\xi_{1}-\eta_{1}} \\
& +A_{\ddagger}^{-1}(\xi) \sum_{m, n} c_{m, n}(a) \int_{-\infty}^{+\infty}\left(\xi_{1}-\eta_{1}\right)^{m}\left(A_{=}^{-1} \widetilde{V}\right)_{\xi_{2}}^{(n)}\left(\eta_{1}, \xi_{2}\right) d \eta_{1}
\end{aligned}
$$

assuming $\widetilde{V} \in S\left(\mathbf{R}^{2}\right), A_{=}^{-1} \widetilde{V}$ means the function $A_{=}^{-1}(\xi) \widetilde{V}(\xi)$.

Proof We need to apply Theorem 2 and to recall correlations between distributions and pseudo-differential operators. It proves the theorem.

Remark 4 The reader can easily write an analog of Theorem 3 corresponding to a rough decomposition. 


\section{Conclusion}

It was shown that the solution of equation (2) for a smooth enough right-hand side $v$ can be represented in the form (10). It shows that in this series the first summand belongs to the space $H_{s}\left(C_{+}^{a}\right)$ only. Secondary summands can be useful for certain special situations related to some additional properties of the right-hand side $v$.

\section{Acknowledgements}

The author thanks the anonymous referee for making several helpful corrections and suggestions.

Funding

Not applicable.

\section{Abbreviations}

Not applicable.

\section{Availability of data and materials}

Not applicable.

Ethics approval and consent to participate

Not applicable.

\section{Competing interests}

The author declares that he has no competing interests.

\section{Consent for publication}

Not applicable.

\section{Authors' contributions}

The author read and approved the final manuscript.

\section{Endnote}

a The first equality is proved by Vladimirov $[20,21]$ in his studies of analytic functions in radial tube domains.

\section{Publisher's Note}

Springer Nature remains neutral with regard to jurisdictional claims in published maps and institutional affiliations.

Received: 12 December 2016 Accepted: 30 July 2017 Published online: 15 August 2017

\section{References}

1. Milkhin, S, Prößdorf, S: Singular Integral Operators. Akademie Verlag, Berlin (1986)

2. Eskin, G: Boundary Value Problems for Elliptic Pseudodifferential Equations. Am. Math. Soc., Providence (1981)

3. Vasil'ev, VB: Wave Factorization of Elliptic Symbols: Theory and Applications. Kluwer Academic, Dordrecht (2000)

4. Vasilyev, VB: Elliptic equations and boundary value problems in non-smooth domains. In: Rodino, L, Wong, MW, Zhu, HT (eds.) Pseudo Differential Operators: Analysis, Applications and Computations. Ball, JA, Dym, H, Kaashoek, MA, Langer, H, Tretter, C (series eds.) Operator Theory: Advances and Applications, vol. 213, pp. 105-121. Birkhäuser, Basel (2011)

5. Vasilyev, VB: New constructions in the theory of elliptic boundary value problems. In: Constanda, C, Kirsch, A (eds.) Integral Methods in Science and Engineering. Theoretical and Computational Advances, pp. 629-641. Birkhäuser, Basel (2015)

6. Vasilyev, VB: Asymptotical analysis of singularities for pseudo differential equations in canonical non-smooth domains. In: Constanda, C, Harris, PE (eds.) Integral Methods in Science and Engineering. Computational and Analytic Aspects, pp. 379-390. Birkhäuser, Boston (2011)

7. Vasilyev, VB: Some problems of pseudo-differential operators theory. Mat. Visn. Nauk. Tov. Im. Shevchenka 10, 219-226 (2013)

8. Kozlov, VA, Mazya, VG, Rossmann, J: Elliptic Boundary Value Problems in Domains with Point Singularities. Am. Math. Soc., Providence (1997)

9. Mazya, V, Rossmann, J: Elliptic Equations in Polyhedral Domains. Am. Math. Soc., Providence (2010)

10. Plamenevskii, B: Solvability of algebras of pseudodifferential operators with piecewise smooth coefficients on smooth manifolds. St. Petersburg Math. J. 21, 317-351 (2010)

11. Schulze, B-W: Pseudo-Differential Boundary Value Problems, Conical Singularities and Asymptotics. Akademie Verlag, Berlin (1994)

12. Melrose, RB: Pseudodofferential operators, corners and singular limits. In: Satake, I (ed.) Proceedings of the International Congress of Mathematicians, Kyoto, Japan, 21-29 August 1990, pp. 217-234. Springer, Berlin (1991)

13. Melrose, RB, Piazza, P: Analytic k-theory on manifolds with corners. Adv. Math. 92, 1-26 (1992)

14. Kottke, C, Melrose, RB: Generalized blow-up of corners and fiber products. Trans. Am. Math. Soc. 367, 651-705 (2015)

15. Hofmann, S, Mitrea, M, Taylor, ME: Symbol calculus for operators of layer potential type on Lipschitz surfaces with vmo normals, and related pseudodifferential operator calculus. Anal. PDE 8, 115-181 (2015) 
16. Mazzurato, A, Nistor, V: Well-posedness and regularity for the elasticity equation with mixed boundary conditions on polyhedral domains and domains with cracks. Arch. Ration. Mech. Anal. 195, 25-73 (2010)

17. Costabel, M, Dauge, M, Nicaise, S: Analytic regularity for linear elliptic systems in polygons and polyhedra. Math. Models Methods Appl. Sci. 22, 1250015 (2012)

18. Costabel, M, Dauge, M, Nicaise, S: Singularities of eddy current problems. ESAIM: Math. Model. Numer. Anal. 37, 807-831 (2003)

19. Mazzeo, R, Melrose, RB: Pseudodifferential operators on manifolds with fibred boundaries. Asian J. Math. 2, 833-866 (1998)

20. Vladimirov, VS: Equations of Mathematical Physics. Dekker, New York (1971)

21. Vladimirov, VS: Methods of the Theory of Generalized Functions. Taylor \& Francis, London (2002)

Submit your manuscript to a SpringerOpen ${ }^{\circ}$ journal and benefit from:

- Convenient online submission

- Rigorous peer review

- Open access: articles freely available online

- High visibility within the field

- Retaining the copyright to your article

Submit your next manuscript at $\gg$ springeropen.com 